\title{
Metodologia para criar objetos de aprendizagem em Matemática usando combinação de ferramentas de autoria
}

\author{
Maria Lucia Pozzatti Flôres ${ }^{1}$, Liane Margarida Rockenbach Tarouco ${ }^{2}$, Eliseo \\ Berni Reategui ${ }^{3}$
}

${ }^{1}$ Doutora pelo Programa de Pós Graduação em Informática na Educação (PGIE) Universidade Federal do Rio Grande do Sul (UFRGS) - Brasil e prof ${ }^{a}$. Universidade Federal do Pampa (UNIPAMPA)

${ }^{2}$ Prof $^{\mathrm{a}}$. Dr ${ }^{\mathrm{a}}$. PGIE/UFRGS - Brasil, professora orientadora

${ }^{3}$ Prof. Dr. PGIE/UFRGS - Brasil, professor coorientador mlflores@terra.com.br liane@penta.ufrgs.br eliseoreategui@gmail.com

\begin{abstract}
This article describes a methodology to create new learning objects (LO) from the reuse of $L O$ using a combination of authoring tools. Shows its validation in a training course for teachers of public schools on the construction of the LO using proposed methodology. At the end of training, course participants answered questionnaires about the constructed objects. These data were statistically analyzed, concluding that teachers with little knowledge of technology, can learn to use and combine authoring tools to create contextualized $L O$.
\end{abstract}

Keywords: reusable learning objects. Contextualized learning objects. Authoring tool.

Resumo Este artigo descreve uma metodologia para criar novos objetos de aprendizagem (OA) a partir da reutilização de OAs usando a combinação de ferramentas de autoria. Mostra a sua validação em um curso de capacitação para professores de Escolas Públicas sobre a construção de OAs utilizando a metodologia proposta. Ao final da capacitação, os participantes do curso responderam questionários sobre os objetos construídos. Esses dados foram analisados estatisticamente, concluindo que os professores, com pouco conhecimento de tecnologia, podem aprender a usar e combinar as ferramentas de autoria para criar OAs contextualizados.

Palavras chave: objetos de aprendizagem reutilizáveis. Objetos de aprendizagem contextualizados. Ferramenta de autoria.

\section{Introdução}

O aluno, ao chegar à escola, não está desprovido de "saberes" e o professor, para ensinar conceitos matemáticos, deve contextualizar a partir desses "saberes" criando condições de problematização. Assim, os alunos serão parte da construção desse conhecimento, o qual será significativo para estes estudantes, pois foi construído e vivenciado por eles. Esse novo conhecimento se integra ao saber pré-existente, modificando-o e sendo por ele modificado [Ausubel 1968].

Os Parâmetros Curriculares Nacionais (PCNs) [Brasil 2001] afirmam que as experiências escolares com o computador têm mostrado que seu uso efetivo pode levar ao estabelecimento de uma nova relação professor-aluno, marcado por uma maior proximidade, interação e colaboração. Com o advento da internet, o professor pode construir OAs contextualizados, com os quais o aluno pode interagir e descobrir, através 
da experiência, não apenas novos conhecimentos, mas também formas de utilizar os conhecimentos aprendidos para resolver problemas matemáticos.

Os computadores podem ser usados nas aulas de Matemática como meio para desenvolver a autonomia pelo uso de software que possibilitam pensar, refletir e criar soluções e como ferramenta para realizar certas atividades. A construção do eixo de simetria de uma parábola, por exemplo, pode ser realizada com a régua, contudo o uso do software Cabri-Géomètre permite, de forma rápida o seu desenho e o estudo de suas propriedades. Softwares como o Cabri-Géomètre, o Winplot, Modellus, Winmat, entre outros, são programas computacionais que apoiam a aprendizagem de Matemática, porém o uso desses softwares necessita de uma estrutura para a formação de professores que os habilite a produzir OAs.

O trabalho de capacitação de professores pode ser um caminho para que os mesmos se sintam encorajados a criar OAs que apoiem a aprendizagem de Matemática. Um curso de poucas horas, todavia, não é suficiente para habilitar um professor a desempenhar o seu papel de mediador na construção do conhecimento matemático usando a tecnologia da informação. Para minimizar esse problema, neste trabalho é proposta uma metodologia para criar e principalmente reusar OAs, utilizando combinação de ferramentas de autoria, as quais permitem ao professor, com pouco conhecimento em informática, manipular, desenvolver e usar OAs.

As ferramentas de autoria permitem a criação de material educacional digital sem que o próprio professor seja um programador, usando estruturas e procedimentos já programados.

Uma vantagem de trabalhar com o reuso de objetos é que diminui o esforço de desenvolvimento, pois permite continuar usando recursos educacionais, sem extenso pré-projeto ou recodificação, o que garante uma economia de tempo e custo para a produção.

Este artigo descreve o OA como recurso de aprendizagem; as teorias que deram subsídio a esta investigação; a metodologia para criar OAs, que prezem estratégias interativas, na qual o aluno tenha participação ativa; e sua validação em um curso de formação de professores para criar OAs com a metodologia proposta.

\section{Objeto de aprendizagem como recurso de aprendizagem}

Objeto de Aprendizagem (OA) é definido como "qualquer recurso digital que pode ser reutilizado para apoiar a aprendizagem" [Wiley 2000]. Normalmente, eles são criados em pequenos módulos que podem ser reusados em diferentes contextos. Eles podem ser contextualizados de maneira similar ao ambiente do mundo real, podendo ser uma única atividade ou um conjunto de estratégias e atividades elaboradas para promover a aprendizagem. Essas características são contempladas quando o OA segue uma padronização de parâmetros. A padronização permite que um ambiente virtual de aprendizagem possa usar objetos de diversos desenvolvedores sem conflito, combinando as possibilidades mais convenientes com o objetivo educacional. A adoção de padrões possibilita uma uniformização, na produção do OA.

É importante observar que as atividades dos OAs devem ser usadas com a finalidade de desenvolver a cognição, e, nesse sentido, precisam ser cuidadosamente planejadas para que a partir delas seja possível construir conhecimentos, desenvolver capacidades, habilidades e competências. Ao manipular as informações na tela, o aluno interage com o computador e se torna o autor e co-autor da construção de seu conhecimento.

\section{Teorias de aprendizagem que suportam esta proposta}


A metodologia proposta se apoiou no Ciclo de Aprendizagem de Kolb (1984), bem como nas recomendações de Wiley (2000) e Gagné (1987), descritas abaixo.

\subsection{Ciclo de Kolb}

Kolb (1984) afirma que a aprendizagem é "o processo pelo qual o conhecimento é criado através da transformação da experiência", por isso, o Ciclo de Kolb é composto por quatro etapas consecutivas: experiência concreta, observação reflexiva, conceituação abstrata e experimentação ativa.

Na visão de Kolb (1984), a experiência é central para o desenvolvimento. Faz parte de um processo dialético e ininterrupto de aprendizagem, presente permanentemente ao longo da vida do indivíduo. As experiências de aprendizagem levam ao desenvolvimento, porque se dirigem a uma meta, um propósito específico de aprendizado.

A transformação da experiência em conhecimento pode ser descrita como um processo pelo qual o indivíduo reflete sobre sua experiência e daí emerge novas aprendizagens. Portanto, ela pode ser definida como um processo que inicia com a experiência, seguida pela reflexão, discussão, análise e avaliação da experiência. Raramente, aprende-se da experiência, a menos que ela seja avaliada, e seja concebido o significado em termos de metas, objetivos, ambições e expectativas. Desses processos surgem os insights, as descobertas e o entendimento. Cada parte do processo assume seu lugar e a experiência toma significado e forma, somando em relação a outras experiências. Isso é então, conceituado, sintetizado e integrado ao sistema de construção do indivíduo, que lhe impõe o mundo pelo qual ele vê, percebe, categoriza, avalia e busca experiência.

\subsection{O sequenciamento de instruções}

O sequenciamento de instrução é recomendado principalmente na área das Ciências Exatas, na qual o aluno segue uma lógica de raciocínio para aprender um novo conceito. Para Merril (1999), a aprendizagem é facilitada caso se apresente aos alunos “organizadores estruturantes e ideias ancoráveis”. As instruções devem ser organizadas a partir de eixos estruturados em torno de uma ideia chave, de um tema.

Para que a aprendizagem ocorra, as atividades precisam envolver o aluno, motivando-o na busca do significado de algum conceito. As atividades devem estar estruturadas em torno de um tema, para que o aluno não desvie sua atenção, não se disperse em seus estudos.

A Metodologia proposta seguirá as recomendações de Wiley (2000) e de Gagné (1987) para estruturar as atividades de aprendizagem, as quais serão estudas a seguir.

\subsubsection{Proposta de Wiley}

Wiley (2000) afirma que o objetivo das teorias instrutivas de um OA é orientar o sequenciamento da instrução. Para isso, ele seguiu a teoria da elaboração de Reigeluth (1999), a qual ajuda os usuários a "selecionar e arranjar os conteúdos em sequência de uma forma a aperfeiçoá-los". Nessa teoria, a instrução deve ser organizada em ordem crescente de complexidade, deve começar pelos conceitos mais gerais e abrangentes que o aluno ainda não tem e, gradativamente, avançar para os conceitos mais restritos e complexos. Por exemplo, para ensinar uma tarefa, primeiro é apresentada a versão mais simples; posteriormente serão adicionadas lições mais complexas até que toda a tarefa seja ensinada. Em cada aula, o aluno deve ser lembrado de todas as versões vistas.

\subsubsection{Proposta de Gagné}

Gagné (1987) sugeriu que a aprendizagem de tarefas para habilidades intelectuais pode ser organizada em uma hierarquia de acordo com a complexidade. $\mathrm{O}$ principal significado da hierarquia é identificar as condições prévias (pré-requisitos) que 
devem ser seguidas para facilitar a aprendizagem em cada nível. A hierarquia fornece uma base para o sequenciamento da instrução. Pela proposta de Gagné (1987), o desenvolvimento de estratégias instrucionais é a sequência de nove "eventos" descritos no Quadro 1

Quadro 1 - Desenvolvimento de estratégias de ensino

\begin{tabular}{|l|l|}
\hline \multicolumn{1}{|c|}{ Componete } & \multicolumn{1}{|c|}{ Descrição } \\
\hline 1. Chamar a atenção & $\begin{array}{l}\text { Pode ser obtido fazendo uma pergunta provocativa } \\
\text { ou apresentando um fato interessante, ou ainda, } \\
\text { apresentando um problema de interesse imediato } \\
\text { para o grupo. }\end{array}$ \\
\hline 2. Descrever os objetivos & $\begin{array}{l}\text { Mostrar o que o aluno vai aprender e como ele vai } \\
\text { poder utilizar o novo conhecimento. }\end{array}$ \\
\hline $\begin{array}{l}\text { 3. Estimular a conexão com o conhecimento } \\
\text { anterior }\end{array}$ & $\begin{array}{l}\text { Explicitar a relação entre o novo e os conceitos já } \\
\text { adquiridos. }\end{array}$ \\
\hline 4. Apresentar o material a ser aprendido & $\begin{array}{l}\text { Apresentar na forma de gráficos, textos, } \\
\text { simulaçôs. Nesta fase, ocorre o momento } \\
\text { essencial da aprendizagem e é quando a } \\
\text { informação entra na memória do aluno. }\end{array}$ \\
\hline 5. Orientar a aprendizagem & $\begin{array}{l}\text { Ajudar o aluno a adquirir capacidades particulares, } \\
\text { especificadas nos objetivos, através da } \\
\text { apresentação de exemplos, estudos de caso, } \\
\text { representações gráficas, material complementar. }\end{array}$ \\
\hline 6. Propiciar desempenho & $\begin{array}{l}\text { Estimular o aluno a desenvolver alguma ação que o } \\
\text { faça mostrar se adquiriu a capacidade desejada, } \\
\text { çiando situações e oferecendo condições para a } \\
\text { aplicação do novo conhecimento. }\end{array}$ \\
\hline 7. Dar feedback ou fazer a realimentação & $\begin{array}{l}\text { Informar o aluno se a atividade desenvolvida por } \\
\text { ele atingiu os objetivos esperados ou não. Quanto } \\
\text { mais detalhada e informativa for essa descrição, } \\
\text { mais fácil é para o aluno identificar os pontos que } \\
\text { precisa melhorar. }\end{array}$ \\
\hline 8. Avaliar & $\begin{array}{l}\text { Verificar se o aluno atingiu os objetivos desejados, } \\
\text { através de testes, verificando o grau de assimilação } \\
\text { do novo conhecimento. }\end{array}$ \\
\hline $\begin{array}{l}\text { 9. Aumentar a retenção e facilitar a transferência } \\
\text { do conhecimento }\end{array}$ & $\begin{array}{l}\text { Disponibilizar ao aluno exercícios de aplicação e e } \\
\text { resolução de problemas. }\end{array}$ \\
\hline
\end{tabular}

\section{Metodologia proposta}

Essa metodologia tem suas pilastras em quatro elementos: planejamento pedagógico; planejamento tecnológico; desenvolvimento do(s) OAs e planejamento das estratégias para aplicação, as quais são mostradas na Figura 1.

\subsection{Planejamento pedagógico}

É compreendido pelos objetivos, a contextualização, os pré-requisitos e a atuação dos participantes.

Objetivos educacionais - nessa etapa são definidos os objetivos do OA com relação ao que se espera em termos de aprendizagem. Esses objetivos devem mostrar o que o aluno vai aprender e como ele vai poder utilizar o novo conhecimento, explicitando a relação entre o novo conhecimento e os conhecimentos já adquiridos. Um OA pode auxiliar na construção do conhecimento se for capaz de servir de ponte entre o conhecimento que o aluno já possui, e o conhecimento novo, corroborando, assim, com a Teoria dos Campos Conceituais de Vergnaud (1994), a qual afirma que cada conceito é constituído a partir de sua participação em uma rede conceitual mais ampla.

Contextualização - está relacionada às atividades e suas aplicações no dia a dia, ou em outras ciências, mas que sejam de domínio dos alunos. De um modo geral, a 
contextualização demanda que se estabeleça qual a relação entre a atividade e situações do dia a dia destes alunos. Pode-se colocar uma situação problema e desafiar os alunos a resolvê-la, ou solicitar aos alunos que estudem temas presentes em reportagens de jornais. Portanto, com relação ao OA, este deve ser criado a partir de um problema matemático dentro do contexto da realidade do aluno.

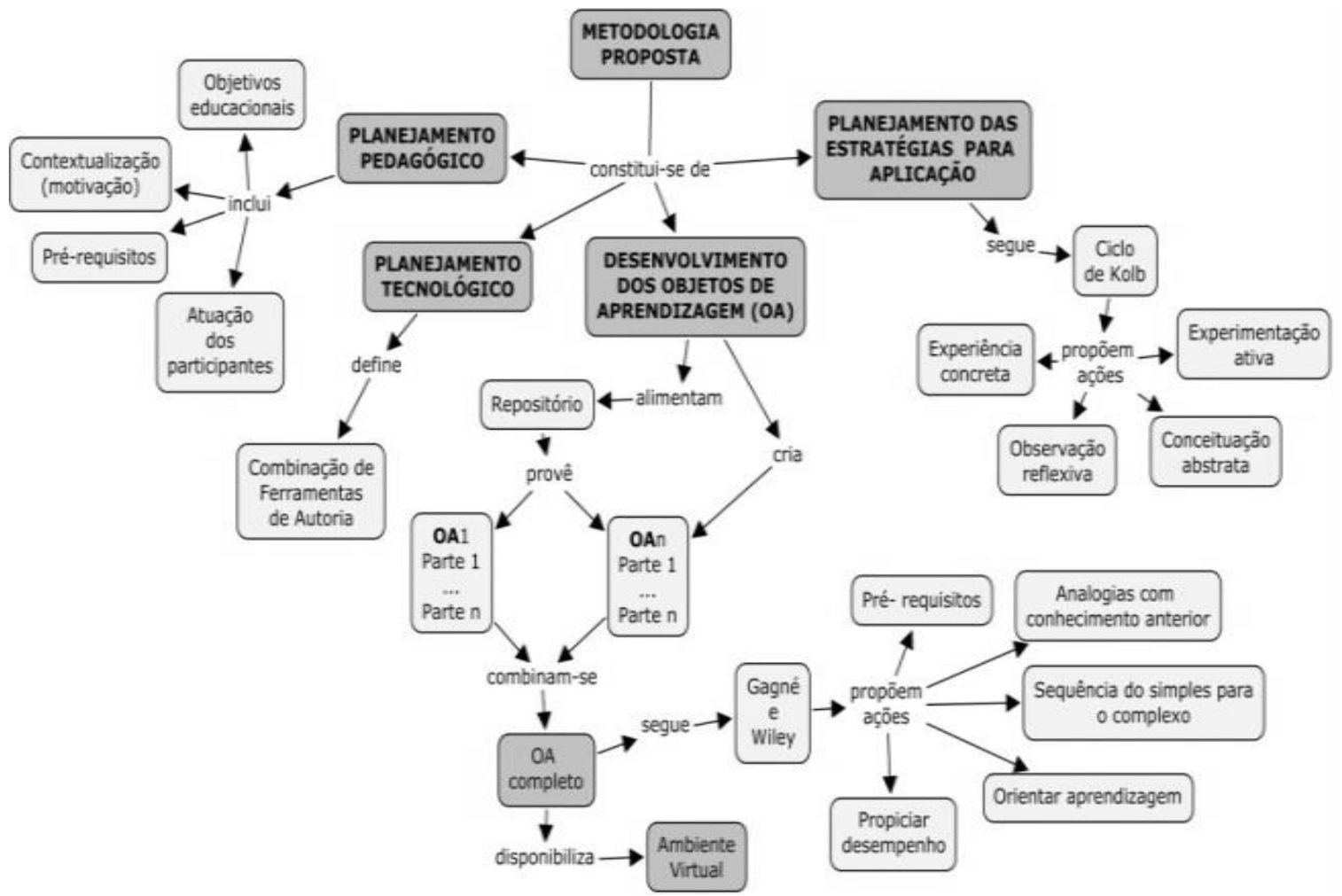

Figura 1 - Metodologia proposta

Pré-requisitos - tratam das condições para o aprendizado. No OA devem estar listados os pré-requisitos necessários para o aprendizado da atividade proposta, bem como a atuação do aluno em relação ao objeto.

Atuação dos participantes - trata das expectativas na relação da atuação dos participantes. A definição de papéis, direitos e deveres de cada agente e a organização do tempo e do espaço.

\subsection{Planejamento tecnológico}

O planejamento tecnológico trata da seleção das técnicas, dos procedimentos e dos recursos informáticos a serem utilizados na atividade.

Seleção das técnicas - decidir que tipo de atividade será usado no OA (resolução de problemas; estudo de caso; ensino por projetos; relato de experiências).

Seleção dos procedimentos - decidir a sequência das ações a serem seguidas para resolver um problema ou efetuar uma tarefa.

Recursos informáticos - propõe-se o uso de ferramentas de autoria, as quais favorecem o professor que não tem profundo conhecimento da tecnologia para criar os OAs. Uma combinação de ferramentas de autoria pode ser empregada, dependendo dos objetivos a serem alcançados e da ênfase dada às atividades previamente estabelecidas. Por exemplo, pode-se criar uma atividade usando uma determinada ferramenta e, em seguida, empregar outra ferramenta para agregar a essa atividade outros recursos, como vídeo, simulações, texto, som, etc. As ferramentas usadas neste projeto foram o eXeLerning e o GeoGebra, que permitem combinar outros OAs com módulos acabados 
disponíveis em repositórios, sendo que o GeoGebra foi inserido no eXe através do iDevice Applet Java.

A navegação no OA pode ser sequencial, de modo que o aluno possa evoluir do raciocínio simples para o complexo. No entanto, as ferramentas de autoria proporcionam menus de navegação que podem levar o aluno diretamente a um ponto desejado. O objeto também poderá ser padronizado para ser disponibilizado em diferentes plataformas computacionais.

\subsection{Desenvolvimento do(s) OA(s)}

O projetista pode fazer um roteiro da sequência de todas as atividades, facilitando assim a construção do objeto. O objeto será construido através das ações:

Seleção de OAs a partir de repositórios - a partir da definição dos objetivos educacionais o professor pode selecionar OAs prontos ou parte dele, encontrados em repositórios, para compor o objeto final e esses objetos serão aglutinados através de uma ferramenta de autoria.

Criar parte do OA - se for necessário, o professor poderá criar um novo objeto, ou parte dele, usando a combinação de ferramentas de autoria. Isto é, ele cria parte do objeto em uma determinada ferramenta e importa esse objeto para outra ferramenta, a qual aglutina todos os objetos menores, transformando em um OA final mais completo e consistente.

OA Completo - segue a proposta de Gagné (1987) e Wiley (2000) que propõem ações: listar os pré-requisitos; explicitar a relação entre o conhecimento novo e os já adquiridos; informar o aluno se a atividade desenvolvida por ele atingiu os objetivos esperados; orientar a aprendizagem; propiciar desempenho. O OA tem as atividades organizadas em uma forma hierárquica de crescente complexidade, a qual identifica os pré-requisitos que devem ser seguidos para facilitar a aprendizagem em cada nível. Essa sequencia das atividades afeta a maneira da informação ser processada e como ocorre a aprendizagem.

Para desenvolver uma atividade, o professor pode também usar diferentes sugestões de atividades. Por exemplo, as proposições contidas no link "Sugestões de Aulas" do Espaço Escola do Portal do Professor, no site do MEC, disponível no endereço http://portaldoprofessor.mec.gov.br , que contém algumas sugestões de aulas para serem desenvolvidas nos Laboratórios de Informática Educativa das escolas.

Esses objetos podem ser padronizados para facilitar a utilização dos componentes desenvolvidos por outros criadores de OAs, aumentando a durabilidade do resultado do esforço de desenvolvimento.

\subsection{Planejamento das estratégias para aplicação}

O planejamento das estratégias inclui o delineamento de ações didáticas para uma determinada situação de aprendizagem. Elas correspondem a um plano, que se constrói e reconstrói através de processos didáticos, que seguem o ciclo de Kolb (1984), e pressupõe que o processo humano de aprendizagem é composto por quatro etapas consecutivas:

Experiência concreta - o OA fornece atividade, com a qual o aluno se envolve em vivências concretas.

Observação reflexiva - o aluno realiza observações e reflexões sobre a experiência.

Conceituação abstrata - o aluno aprende com a experiência e elabora conceitos abstratos.

Experimentação ativa - o aluno experimenta o que aprendeu. Ele faz generalizações, que permitem um novo contato com a realidade com o objetivo de testar os resultados e 
suas implicações em novas situações por meio de experimentação ativa, levando-o ao início do ciclo para novas vivências concretas e assim sucessivamente.

\section{Curso de capacitação}

Para validar essa metodologia foi proposto um curso de Capacitação aos professores municipais e estaduais da disciplina de Matemática com o objetivo de ensinar a construção de OAs contextualizados e completos. Esse curso foi organizado para ter cinco encontros, cada um com uma duração de 4 horas, o que totalizou 20 horas. Os três primeiros encontros foram realizados no Laboratório da Ulbra, Campus de Santa Maria, nos quais foram desenvolvidos os conhecimentos sobre: OAs e ferramentas de autoria através da experimentação e reflexão mediadas pelo professor, usando os seguintes recursos: computador, Datashow, ferramenta de autoria eXeLearning e software GeoGebra. O $4^{\mathrm{a}}$ encontro foi virtual, no qual o participante desenvolveu um OA de Matemática. O $5^{\mathrm{a}}$ encontro foi presencial, através de um seminário, no qual o participante apresentou o seu OA construído e respondeu dois questionários, um sobre o processo de construção de seu OA e o outro analisando se os OAs de seus pares seguiram a metodologia proposta. Ao final da capacitação foi aplicado um questionário avaliando o próprio curso.

Essa capacitação foi oferecida, gratuitamente, mas em contrapartida, os participantes disponibilizaram seus OAs para a comunidade como um todo, dentro da estratégia de conteúdo aberto e livre. Na população de professores municipais e estaduais de Matemática, foi selecionada uma amostra aleatória de vinte professores para integrar a primeira turma. Dessa amostra, treze professores participaram do experimento até o final e construíram onze objetos, pois quatro professores trabalharam em dupla, formando duas diferentes duplas. Os nove professores restantes criaram seus objetos de aprendizagem individualmente. Cada participante (individual ou em dupla) criou o seu OA usando a metodologia proposta pela autora. Desses objetos, três foram descartados, pois os participantes o "perderam" na hora de salvar e postar no ambiente virtual de aprendizagem Moodle da Ulbra-SM, mas todos os treze participantes responderam os questionários propostos ao final da capacitação.

\subsection{OAs produzidos pelos participantes}

Os objetos construídos e postados no ambiente Moodle da Ulbra Santa Maria estão resumidos a seguir:

- Latitude com GeoGebra: objeto de conteúdo de Geografia, mas usa a Matemática ao usar a Geometria para a localização. Nesse objeto o professor combina a ferramenta eXe com o GeoGebra e contextualiza a latitude e a longitude de vários pontos geográficos.

- Questões de Geometria Plana: objeto que associa materiais de Geometria plana no eXe, mostrando a construção da Bandeira do Brasil utilizando o software GeoGebra e desafiando o aluno a fazer o mesmo.

- Super - Herói: objeto criado com a ferramenta eXe, no qual é feito um levantamento do tipo de super-herói preferido pelos alunos e mostrando o resultado em um gráfico.

- Parábola: explora, no GeoGebra, o comportamento da parábola em relação a sua concavidade e usa o eXe para aglutinar os diferentes recursos.

- Localização dos pontos Cardeais: trabalha com pontos cardeais e colaterais no eXe e mostra a localização dos jogadores no campo de futebol usando GeoGebra.

- Função Quadrática: utiliza o eXe para mostrar exemplos de função quadrática e colocar o aluno dentro do GeoGebra para que ele construa uma parábola. 
- Triângulo Retângulo: apresenta, no GeoGebra, os conceitos do seno, cosseno e tangente do triângulo retângulo, mostrando também como medir a altura de uma árvore usando essas medidas. Aglutina todos os recursos no eXe.

- Quadrado da Soma: mostra geometricamente, no GeoGebra, o quadrado da soma de dois termos. Elabora várias atividades e as aglutina no eXe.

\subsection{Análises estatísticas dos dados coletados}

Com os dados coletados nos questionários aplicados ao final da Capacitação foram realizados testes de hipótese da proporção, ao nível de significância de 95\%, e a variável de teste z (normal padronizada), para testar as hipóteses:

(a) Os professores de Matemática podem aprender facilmente usar e combinar as ferramentas de autoria para criar OAs de acordo com a metodologia proposta.

(b) Os OAs criados com a metodologia proposta atendem critérios de sequenciamento e de reflexão.

Como o teste das proporções tem restrições quando as frequências obtidas (fn) forem menores que 5, que é o caso das frequências das respostas "discordam", então foram também realizados testes exato de Fisher, que é adequado para pequenas amostras, a partir dos mesmos dados amostrais e nível de significância do teste da proporção, com o objetivo de confirmar os resultados destes.

\subsubsection{Resultados do questionário sobre o processo de criação dos Objetos de Aprendizagem}

A partir dos dados recolhidos do primeiro questionário respondidos pelos treze participantes finais e agrupando as respostas "concordo fortemente e concordo parcialmente" como uma resposta positiva, e ainda, "discordo parcialmente e fortemente" como uma resposta negativa foi elaborado um teste de significância para a igualdade de duas proporções, testando a hipótese (a) e obtendo-se como resultado que os professores aprendem rapidamente as ferramentas de autoria para construir OAs. Esse resultado foi também confirmado pelo teste exato de Fisher, com os mesmos dados anteriores.

Contudo, três professores registraram em seus questionários que encontraram dificuldade ao usar o iDevice Applet Java para importar o objeto criado no GeoGebra para o eXe, apesar de concluírem seus objetos. Esses participantes só conseguiram importar o objeto criado no GeoGebra para o eXe na segunda tentativa de importação.

\subsubsection{Resultados do questionário sobre a análise dos objetos apresentados}

A cada OA apresentado foram sorteados, com reposição, cinco participantes para analisar e responder o questionário, totalizando quarenta questionários respondidos. Agrupando as respostas "concordo fortemente e parcialmente" como uma resposta positiva e, ainda, "discordo parcialmente e fortemente" como uma resposta negativa elaborou-se um teste de significância para a igualdade de duas proporções, testando a hipótese (b) e obteve-se como resultado que os OAs atendem critérios de sequênciamento e de experimento.

Apesar de a amostra estudada ser considerada grande, foi realizado o teste de Fisher, pois algumas frequências das respostas "discordam" foram menores do que 5 (cinco), confirmando o resultado anterior.

\subsection{Resultados sobre o questionário para analisar o curso de capacitação}

Em relação ao curso de Capacitação foi aplicado um questionário para os participantes avaliá-lo, cujos resultados são:

$75 \%$ dos participantes perceberam que a integração dos conteúdos, eXe, GeoGebra e objetos de aprendizagem se deu na medida certa, sendo que $62 \%$ dos 
participantes acharam conteúdo em demasia, quando comparado a um curso "tradicional". A combinação de ferramentas de autoria e, principalmente, a quantidade de recursos foi aprovada pelos participantes, pois não se julgaram limitados e tiveram a liberdade para a criação do objeto.

$75 \%$ consideraram que a oportunidade para colocar a experimentação, a reflexão e a sequenciação do conteúdo na criação de OA, estava na dose certa, e com mais oportunidade quando comparada a um curso "tradicional".

$88 \%$ dos participantes responderam que aprenderam e gostaram muito do curso; sendo que todos os participantes o recomendam para outras pessoas.

\section{Conclusão}

Os PCNs [Brasil 2001] sugerem que as experiências escolares com o computador têm sido positivas e o seu uso efetivo pode levar ao estabelecimento de uma nova relação professor-aluno, marcada por uma maior proximidade, interação e colaboração. Nesse contexto, o professor pode investir na criação de OAs para a construção do conhecimento matemático de seu aluno, tornando suas aulas mais atraentes. Com o uso de OAs o aluno pode construir várias estratégias sobre determinada atividade, obtendo realimentação do computador que o auxilia na correção dessas estratégias, podendo diminuir assim, as dificuldades do aprendizado de Matemática.

Para criar OAs o professor de Matemática pode reusar OAs com a combinação de ferramentas de autoria, organizados conforme uma sequência de atividades aconselhável, combinado ou não com outros objetos, de forma a ser capaz de atender as necessidades de aprendizagem de determinado conceito matemático. A combinação e os novos objetos devem seguir o sequenciamento de instrução proposta por Wiley (2000) e Gagné (1987), isto é, a instrução pode ser organizada em uma hierarquia de acordo com a complexidade, a qual identifica os pré-requisitos que devem ser seguidos para facilitar a aprendizagem em cada nível. Esses OAs também seguiram a proposta do Ciclo de Kolb (1994) para que a aprendizagem seja realmente efetivada. Assim, eles devem fornecer atividades em que o aluno se envolve com vivências concretas, nas quais realiza observações e reflexões sobre seu contato com o mundo, elaborando conceitos abstratos e generalizações que permitem um novo contato com a realidade, com o objetivo de testar os resultados e suas implicações em novas situações por meio da experimentação ativa.

Os OAs de Matemática foram construídos usando a combinação de Ferramentas de Autoria eXe e GeoGebra, pois elas permitem ao professor, com pouco conhecimento em informática, manipular, desenvolver e usar esses OAs. Elas permitem a criação de material educacional digital sem que o próprio professor seja um programador, usando estruturas e procedimentos já programados, reunindo-os, agregando conteúdo e forma de tratamento aos dados que dependem de sua estratégia pedagógica. O professor não precisa ser um programador, mas deve ter conhecimentos básicos de informática e capacitação para o uso dessas ferramentas. Assim, as ferramentas de autoria, aliadas à experiência do professor dão mais condições para que o resultado do OA atenda os objetivos de seu uso como ferramenta de apoio ao processo de ensino aprendizagem.

Uma das vantagens dessa metodologia é que ela trabalha com reuso de objetos, diminuindo assim o esforço de desenvolvimento, pois permite continuar usando recursos educacionais, sem extenso pré-projeto ou recodificação, o que garante uma economia de tempo e custo para a produção. Esses objetos podem ser padronizados para facilitar a utilização dos componentes desenvolvidos por outros criadores de OAs, aumentando a durabilidade do resultado do esforço de desenvolvimento. 
Essa metodologia foi validada através do curso de Capacitação de Professores para ensinar a construção de OAs combinando a ferramenta de autoria eXeLearning e o software GeoGebra. Nesse curso foram construídos OAs com atividades contextualizadas envolvendo o conteúdo de Matemática, os quais foram postados no ambiente virtual de aprendizagem Moodle da ULBRA de Santa Maria. Os participantes do curso responderam três questionários, cujos dados foram analisados estatisticamente, chegando-se a conclusão de que os professores aprendem rapidamente as ferramentas de autoria para construir OAs e os objetos construídos atendem critérios de sequenciamento e de experimento.

Um ponto que deve ser repensado é que a maioria das ferramentas de autorias não possui muita flexibilidade, pois segue os modelos predefinidos pelo ambiente. Isso, por um lado, é uma vantagem, devido ao fato de que nem sempre o professor poderá ter a sua disposição um técnico da área de informática, por outro lado é uma desvantagem, pois o professor fica preso aos modelos da ferramenta.

\section{Referências}

BRASIL- MEC. (2001) Secretaria de Educação Fundamental. Parâmetros curriculares nacionais: matemática. Brasília: MEC/SEF.

eXelearning. Site disponível em: http://exelearning.org/

GAGNÉ, R. (1987) Instructional Technology Foundations. Hillsdale, NJ: Lawrence Erlbaum Assoc.

GeoGebra. Site disponível em: http://www.geogebra.org

KOLB, D. (1997). A gestão e o processo de aprendizagem. In: Starkey, Ken. Como as organizações aprendem. São Paulo: Futura/Zumble.

KOLB, D (1984) Experiential learning: Experience as the source of learning and development. New Jersey: Prentice-Hall.

MERRIL M. D. (1999) Instructional transaction theory (ITT): Instructional design based on knowledge objects. In C. M. Reigeluth (Ed.), Instructional design theories and models: A new paradigm of instructional theory. (pp. 397-424). Hillsdale, NJ: Lawrence Erlbaum Associates.

REIGELUTH C. M.(1999) Instructional design theories and models: A new paradigm of instructional theory. (pp. 5-29). Hillsdale, NJ: Lawrence Erlbaum Associates.

VERGNAUDT, G. (1994). L'enfant, la mathématique et la réalité. Paris: Peter Lang.

WILEY, D. A. (2000). Learning object design and sequencing theory. Doctoral dissertation, Brigham Young University. 